\title{
Interview with Pierre Deligne
}

Martin Raussen and Christian Skau

Pierre Deligne is the recipient of the 2013 Abel Prize of the Norwegian Academy of Science and Letters. This interview was conducted by Martin Raussen and Christian Skau in Oslo in May 2013 in conjunction with the Abel Prize celebration. This article originally appeared in the September 2013 issue of the Newsletter of the European Mathematical Society and is reprinted here with permission of the EMS.

Raussen and Skau: Dear Professor Deligne, first of all we would like to congratulate you as the eleventh recipient of the Abel Prize. It is not only a great honor to be selected as recipient of this prestigious prize, the Abel Prize also carries a cash amount of six million NOK, that is around US\$1,000,000. We are curious to hear what you are planning to do with this money...

Deligne: I feel that this money is not really mine, but it belongs to mathematics. I have a responsibility to use it wisely and not in a wasteful way. The details are not clear yet, but I plan to give part of the money to the two institutions that have been most important to me: the Institut des Hautes Études Scientifiques (IHÉS) in Paris and the Institute for Advanced Study (IAS) in Princeton.

I would also like to give some money to support mathematics in Russia. First to the Department of Mathematics of the Higher School of Economics (HSE). In my opinion, it is one of the best places in Moscow. It is much smaller than the Faculty of Mechanics and Mathematics at the [National Research] University, but has better people. The student body is small; only fifty new students are accepted each year. But they are among the best students. The HSE was created by economists. They have done their best under difficult circumstances. The department of mathematics has been created five years ago, with the help of the Independent University of Moscow. It is giving prestige to the whole HSE. There I think some money could be well used.

Another Russian institution I would like to donate some money to is the Dynasty Foundation, created by the Russian philanthropist Dmitry Zimin. For them, money is most likely not that important. It is rather a way for me to express my

Martin Raussen is associate professor of mathematics at Aalborg University, Denmark. His email address is raussen@math.aau.dk.

Christian Skau is professor of mathematics at the Norwegian University of Science and Technology, Trondheim, Norway. His email address is csk@math . ntnu. no. DOI: http://dx.doi.org/10.1090/noti1081

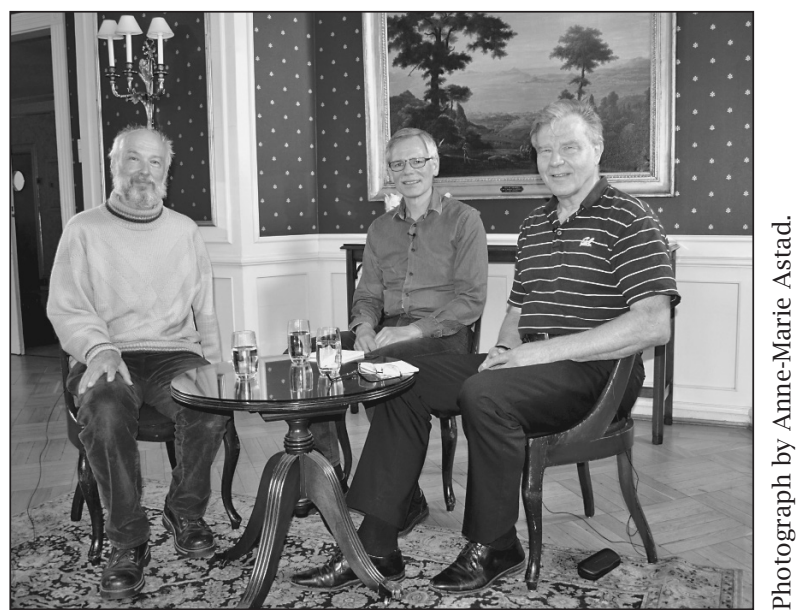

From left to right: Pierre Deligne, Martin Raussen, Christian Skau.

admiration for their work. It is one of the very few foundations in Russia that gives money to science; moreover, they do it in a very good way. They give money to mathematicians, to physicists, and to biologists; especially to young people, and this is crucial in Russia! They also publish books to popularize science. I want to express my admiration for them in a tangible way.

Raussen and Skau: The Abel Prize is certainly not the first important prize in mathematics that you have won. Let us just mention the Fields Medal that you received 35 years ago, the Swedish Crafoord Prize, the Italian Balzan Prize and the Israeli Wolf Prize. How important is it for you, as a mathematician, to win such prestigious prizes? And how important is it for the mathematical community that such prizes exist?

Deligne: For me personally, it is nice to be told that mathematicians I respect find the work I have done interesting. The Fields Medal possibly helped me to be invited to the Institute for Advanced Study. To win prizes gives opportunities, but they have not changed my life.

I think prizes can be very useful when they can serve as a pretext for speaking about mathematics to the general public. I find it particularly nice that 
the Abel Prize is connected with other activities such as competitions directed towards children and the Holmboe Prize for high school teachers. In my experience, good high school teachers are very important for the development of mathematics. I think all these activities are marvellous.

\section{Youth}

Raussen and Skau: You were born in 1944, at the end of the Second World War in Brussels. We are curious to hear about your first mathematical experiences: In what respect were they fostered by your own family or by school? Can you remember some of your first mathematical experiences?

Deligne: I was lucky that my brother was seven years older than me. When I looked at the thermometer and realized that there were positive and negative numbers, he would try to explain to me that minus one times minus one is plus one. That was a big surprise. Later when he was in high school he told me about the second degree equation. When he was at the university he gave me some notes about the third degree equation, and there was a strange formula for solving it. I found it very interesting.

When I was a Boy Scout, I had a stroke of extraordinary good luck. I had a friend there whose father, Monsieur Nijs, was a high school teacher. He helped me in a number of ways; in particular, he gave me my first real mathematical book, namely Set Theory by Bourbaki, which is not an obvious choice to give to a young boy. I was fourteen years old at the time. I spent at least a year digesting that book. I guess I had some other lectures on the side, too.

Having the chance to learn mathematics at one's own rhythm has the benefit that one revives surprises of past centuries. I had already read elsewhere how rational numbers, then real numbers, could be defined starting from the integers. But I remember wondering how integers could be defined from set theory, looking a little ahead in Bourbaki, and admiring how one could first define what it means for two sets to have the "same number of elements", and derive from this the notion of integers. I was also given a book on complex variables by a friend of the family. To see that the story of complex variables was so different from the story of real variables was a big surprise: once differentiable, it is analytic (has a power series expansion), and so on. All those things that you might have found boring at school were giving me a tremendous joy.

Then this teacher, Monsieur Nijs, put me in contact with Professor Jacques Tits at the University of Brussels. I could follow some of his courses and seminars, though I still was in high school.

Raussen and Skau: It is quite amazing to hear that you studied Bourbaki, which is usually considered quite difficult, already at that age. Can you tell us a bit about your formal school education? Was that interesting for you, or were you rather bored?

Deligne: I had an excellent elementary school teacher. I think I learned a lot more in elementary school than I did in high school: how to read, how to write, arithmetic, and much more. I remember how this teacher made an experiment in mathematics that made me think about proofs, surfaces, and lengths. The problem was to compare the surface of a half-sphere with that of the disc with the same radius. To do so, he covered both surfaces with a spiralling rope. The half sphere required twice as much rope. This made me think a lot: how could one measure a surface with a length? How to be sure that the surface of the half sphere was indeed twice that of the disc?

When I was in high school, I liked problems in geometry. Proofs in geometry make sense at that age because surprising statements have not-toodifficult proofs. Once we were past the axioms, I enjoyed very much doing such exercises. I think that geometry is the only part of mathematics where proofs make sense at the high school level. Moreover, writing a proof is another excellent exercise. This does not only concern mathematics, you also have to write in correct French-in my case-in order to argue why things are true. There is a stronger connection between language and mathematics in geometry than for instance in algebra, where you have a set of equations. The logic and the power of language are not so apparent.

Raussen and Skau: You went to the lectures of Jacques Tits when you were only sixteen years old. There is a story that one week you could not attend because you participated in a school trip...?

Deligne: Yes. I was told this story much later. When Tits came to give his lecture he asked: Where is Deligne? When it was explained to him that I was on a school trip, the lecture was postponed to the next week.

Raussen and Skau: He must already have recognized you as a brilliant student. Jacques Tits is also a recipient of the Abel Prize. He received it together with John Griggs Thompson five years ago for his great discoveries in group theory. He was surely an influential teacher for you?

Deligne: Yes; especially in the early years. In teaching, the most important can be what you don't do. For instance, Tits had to explain that the center of a group is an invariant subgroup. He started a proof, then stopped and said in essence: "An invariant subgroup is a subgroup stable by all inner automorphisms. I have been able to define the center. It is hence stable by all symmetries of the data. So it is obvious that it is invariant."

For me, this was a revelation: the power of the idea of symmetry. That Tits did not need to go through a step-by-step proof, but instead could just say that symmetry makes the result obvious, 
has influenced me a lot. I have a very big respect for symmetry, and in almost every one of my papers there is a symmetry-based argument.

Raussen and Skau: Can you remember how Tits discovered your mathematical talent?

Deligne: That I cannot tell, but I think it was Monsieur Nijs who told him to take good care of me. At that time, there were three really active mathematicians in Brussels: apart from Tits himself, Professors Franz Bingen and Lucien Waelbroeck. They organized a seminar with a different subject each year. I attended these seminars, and I learned about different topics such as Banach algebras, which were Waelbroeck's speciality, and algebraic geometry.

Then, I guess, the three of them decided it was time for me to go to Paris. Tits introduced me to Grothendieck and told me to attend his lectures as well as Serre's. That was an excellent advice.

Raussen and Skau: This can be a little surprising to an outsider. Tits being interested in you as a mathematician, one might think that he would try to capture you for his own interests. But he didn't?

Deligne: No. He saw what was best for me and acted accordingly.

\section{Algebraic Geometry}

Raussen and Skau: Before we proceed to your career in Paris, perhaps we should try to explain to the audience what your subject, algebraic geometry, is about.

When Fields medalist Tim Gowers had to explain your research subjects to the audience during the Abel Prize announcement earlier this year, he began by confessing that this was a difficult job for him. It is difficult to show pictures that illustrate the subject, and it is also difficult to explain some simple applications. Could you, nevertheless, try to give us an idea what algebraic geometry is about? Perhaps you can mention some specific problems that connect algebra and geometry with each other.

Deligne: In mathematics, it is always very nice when two different frames of mind come together. Descartes wrote: "La géométrie est l'art de raisonner juste sur des figures fausses (Geometry is the art of correct reasoning on false figures)." "Figures" is plural: it is very important to have various perspectives and to know in which way each is wrong.

In algebraic geometry, you can use intuitions coming both from algebra-where you can manipulate equations-and from geometry, where you can draw pictures. If you picture a circle and consider the equation $x^{2}+y^{2}=1$, different images are evoked in your mind, and you can try to play one against the other. For instance, a wheel is a circle and a wheel turns; it is interesting to see what the analogue is in algebra: an algebraic transformation of $x$ and $y$ maps any solution of $x^{2}+y^{2}=1$ to another. This equation describing a circle is of the second degree. This implies that a circle will have no more than two intersection points with a line. This is a property you also see geometrically, but the algebra gives more. For instance, if the line has a rational equation and one of the intersection points with the circle $x^{2}+y^{2}=1$ has rational coordinates, then the other intersection point will also have rational coordinates.

Algebraic geometry can have arithmetical applications. When you consider polynomial equations, you can use the same expressions in different number systems. For instance, on finite sets on which addition and multiplication are defined, these equations lead to combinatorial questions: you try to count the number of solutions. But you can continue to draw the same pictures, keeping in mind a new way in which the picture is false, and in this way you can use geometrical intuition while looking at combinatorial problems.

I have never really been working at the center of algebraic geometry. I have mostly been interested in all sorts of questions that only touch the area. But algebraic geometry touches many subjects! As soon as a polynomial appears, one can try to think about it geometrically; for example in physics with Feynman integrals, or when you consider an integral of a radical of a polynomial expression. Algebraic geometry can also contribute to the understanding of integer solutions of polynomial equations. You have the old story of elliptic functions: to understand how elliptic integrals behave, the geometrical interpretation is crucial.

Raussen and Skau: Algebraic geometry is one of the main areas in mathematics. Would you say that to learn algebraic geometry requires much more effort than other areas in mathematics, at least for a beginner?

Deligne: I think it's hard to enter the subject because one has to master a number of different tools. To begin with, cohomology is now indispensable. Another reason is that algebraic geometry developed in a succession of stages, each with its own language. First, the Italian school which was a little hazy, as shown by the infamous saying: "In algebraic geometry, a counterexample to a theorem is a useful addition to it." Then Zariski and Weil put things on a better footing. Later Serre and Grothendieck gave it a new language, which is very powerful. In this language of schemes one can express a lot; it covers both arithmetical applications and more geometrical aspects. But it requires time to understand the power of this language. Of course, one needs to know a number of basic theorems, but I don't think that this is the main stumbling block. The most difficult is to understand the power of the language created by Grothendieck and how it relates to our usual geometrical intuition. 


\section{Apprentice in Paris}

Raussen and Skau: When you came to Paris you came in contact with Alexander Grothendieck and Jean-Pierre Serre. Could you tell us about your first impression of these two mathematicians?

Deligne: I was introduced to Grothendieck by Tits during the Bourbaki seminar of November 1964. I was really taken aback. He was a little strange, with his shaved head, a very tall man. We shook hands but did nothing more until I went to Paris a few months later to attend his seminar.

That was really an extraordinary experience. In his way, he was very open and kind. I remember the first lecture I attended. In it, he used the expression "cohomology object" many times. I knew what cohomology was for abelian groups, but I did not know the meaning of "cohomology object". After the lecture I asked him what he meant by this expression. I think that many other mathematicians would have thought that if you didn't know the answer, there wouldn't be any point to speak to you. This was not his reaction at all. Very patiently he told me that if you have a long exact sequence in an abelian category and you look at the kernel of one map, you divide by the image of the previous one and so on... I recognized quickly that I knew about this in a less general context. He was very open to people who were ignorant. I think that you should not ask him the same stupid question three times, but twice was all right.

I was not afraid to ask completely stupid questions, and I have kept this habit until now. When attending a lecture, I usually sit in front of the audience, and if there is something I don't understand, I ask questions even if I would be supposed to know what the answer was.

I was very lucky that Grothendieck asked me to write up talks he had given the previous year. He gave me his notes. I learned many things, both the content of the notes, and also a way of writing mathematics.... This was both in a prosaic way, namely that one should write only on one side of the paper and leave some blank space so he could make comments, but he also insisted that one was not allowed to make any false statement. This is extremely hard. Usually one takes shortcuts; for instance, not keeping track of signs. This would not pass muster with him. Things had to be correct and precise. He told me that my first version of the redaction was much too short, not enough details.... It had to be completely redone. That was very good for me.

Serre had a completely different personality. Grothendieck liked to have things in their natural generality; to have an understanding of the whole story. Serre appreciates this, but he prefers beautiful special cases. He was giving a course at Collège de France on elliptic curves. Here, many different strands come together, including automorphic forms. Serre had a much wider mathematical culture than Grothendieck. In case of need, Grothendieck redid everything for himself, while Serre could tell people to look at this or that in the literature. Grothendieck read extremely little; his contact with classical Italian geometry came basically through Serre and Dieudonné. I think Serre must have explained to him what the Weil conjectures were about and why they were interesting. Serre respected the big constructions Grothendieck worked with, but they were not in his taste. Serre preferred smaller objects with beautiful properties such as modular forms, to understand concrete questions, for instance congruences between coefficients.

Their personalities were very different, but I think that the collaboration between Serre and Grothendieck was very important and it enabled Grothendieck to do some of his work.

Raussen and Skau: You told us that you needed to go to Serre's lectures in order to keep your feet on the ground?

Deligne: Yes, because there was a danger in being swept away in generalities with Grothendieck. In my opinion, he never invented generalities that were fruitless, but Serre told me to look at different topics that all proved to be very important for me.

\section{The Weil Conjectures}

Raussen and Skau: Your most famous result is the proof of the third-and the hardest-of the so-called Weil conjectures. But before talking about your achievement, can you try to explain why the Weil conjectures are so important?

Deligne: There were some previous theorems of Weil about curves in the one-dimensional situation. There are many analogies between algebraic curves over finite fields and the rational numbers. Over the rational numbers, the central question is the Riemann hypothesis. Weil had proved the analogue of the Riemann hypothesis for curves over finite fields, and he had looked at some higher-dimensional situations as well. This was at the time where one started to understand the cohomology of simple algebraic varieties, like the Grassmannians. He saw that some point-counting for objects over finite fields reflected what happened over the complex numbers and the shape of the related space over the complex numbers.

As Weil looked at it, there are two stories hidden in the Weil conjectures. First, why should there be a relation between apparently combinatorial questions and geometric questions over the complex numbers? Second, what is the analogue of the Riemann hypothesis? Two kinds of applications came out of these analogies. The first started with Weil himself: estimates for some arithmetical functions. For me, they are not the most important. Grothendieck's construction of a formalism explaining why there should be a relation between 
the story over the complex numbers, where one can use topology, and the combinatorial story, is more important.

Secondly, algebraic varieties over finite fields admit a canonical endomorphism, the Frobenius. It can be viewed as a symmetry, and this symmetry makes the whole situation very rigid. Then one can transpose this information back into the geometric world over the complex numbers; it yields constraints on what will happen in classical algebraic geometry, and this is used in applications to representation theory and the theory of automorphic forms. It was not obvious at first that there would be such applications, but for me they are the reason why the Weil conjecture is important.

Raussen and Skau: Grothendieck had a program on how to prove the last Weil conjecture, but it didn't work out. Your proof is different. Can you comment on this program? Did it have an influence on the way you proved it?

Deligne: No. I think that the program of Grothendieck was, in a sense, an obstruction to finding the proof, because it made people think in just a certain direction. It would have been more satisfying if one had been able to do the proof following the program, because it would have explained a number of other interesting things as well. But the whole program relied on finding enough algebraic cycles on algebraic varieties, and on this question one has made essentially no progress since the 1970s.

I used a completely different idea. It is inspired by the work of Rankin and his work on automorphic forms. It still has a number of applications, but it did not realize the dream of Grothendieck.

Raussen and Skau: We heard that Grothendieck was glad that the Weil conjecture was proved, of course, but still he was a little disappointed?

Deligne: Yes. And with very good reason. It would have been much nicer if his program had been realized. He did not think that there would be another way to do it. When he heard I had proved it, he felt I must have done this and that, which I hadn't. I think that's the reason for the disappointment.

Raussen and Skau: You have to tell us about the reaction of Serre when he heard about the proof.

Deligne: I wrote him a letter when I did not have a complete proof yet, but a test case was clear. I think he got it just before he had to go to the hospital for an operation of a torn tendon. He told me later that he went into the operation theatre in a euphoric state because he knew now that the proof was roughly done.

Raussen and Skau: Several famous mathematicians have called your proof of the last Weil conjecture a marvel. Can you describe how you got the ideas that led to the proof?

Deligne: I was lucky that I had all the tools needed at my disposal at the same time and that
I understood that those tools would do it. Parts of the proof have since been simplified by Gérard Laumon, and a number of these tools are no longer needed.

At the time, Grothendieck had ideas for putting into a purely algebraic framework the work of Solomon Lefschetz from the 1920s about families of hyperplane sections of an algebraic variety. Of particular interest was a statement of Lefschetz, later proved by William Hodge, the so-called hard Lefschetz theorem. Lefschetz's approach was topological. In contrast to what one might think, if arguments are topological there is a better chance to translate them into abstract algebraic geometry than if they are analytic, such as the proof given by Hodge. Grothendieck asked me to look at the 1924 book L'analysis situs et la géométrie algébrique by Lefschetz. It is a beautiful and very intuitive book, and it contained some of the tools I needed.

I was also interested in automorphic forms. I think it is Serre who told me about an estimate due to Robert Rankin. I looked carefully at it. Rankin was getting some nontrivial estimates for coefficients of modular forms by proving for some related $L$-functions what was needed to apply results of Landau, in which the location of the poles of an $L$-function gave information on the poles of the local factors. I saw that the same tool, in a much less sophisticated way, just using that a sum of squares is positive, could be used here because of the control the work of Grothendieck gave on poles. This was enough. The poles were much easier to understand than the zeros and it was possible to apply Rankin's idea.

I had all these tools at my disposal, but I cannot tell how I put them together.

Raussen and Skau: What is a motive?

Deligne: A surprising fact about algebraic varieties is that they give rise not to one, but to many cohomology theories, among them the $l$-adic theories, one for each prime $l$ different from the characteristic, and in characteristic zero, the algebraic de Rham cohomology. These theories seem to tell the same story, over and over again, each in a different language. The philosophy of motives is that there should exist a universal cohomology theory, with values in a category of motives to be defined, from which all these theories could be derived. For the first cohomology group of a projective nonsingular variety, the Picard variety plays the role of a motivic $H^{1}$ : the Picard variety is an abelian variety, and from it the $H^{1}$ in all available cohomology theories can be derived. In this way, abelian varieties (taken up to isogeny) are a prototype for motives.

A key idea of Grothendieck is that one should not try to define what a motive is. Rather, one should try to define the category of motives. It should be an abelian category with finite dimensional rational vector spaces as Hom groups. 
Crucially, it should admit a tensor product, needed to state a Künneth theorem for the universal cohomology theory, with values in the category of motives.

If only the cohomology of projective nonsingular varieties is considered, one speaks of pure motives. Grothendieck proposed a definition of a category of pure motives and showed that, if the category defined had a number of properties, modeled on those of Hodge structures, the Weil conjectures would follow.

For the proposed definition to be viable, one needs the existence of "enough" algebraic cycles. On this question almost no progress has been made.

\section{A Little Bit about Subsequent Work}

Raussen and Skau: What about your other results? Which of those that you worked on after the proof of the Weil conjecture are you particularly fond of?

Deligne: I like my construction of a so-called mixed Hodge structure on the cohomology of complex algebraic varieties. In its genesis, the philosophy of motives has played a crucial role, even if motives don't appear in the end result. The philosophy suggests that, whenever something can be done in one cohomology theory, it is worthwhile to look for a counterpart in other theories. For projective nonsingular varieties, the role played by the action of Galois is similar to the role played by the Hodge decomposition in the complex case. For instance, the Hodge conjecture, expressed using the Hodge decomposition, has as counterpart the Tate conjecture, expressed using the action of Galois. In the l-adic case, cohomology and the action of Galois remain defined for singular or noncompact varieties.

This forces us to ask: what is the analogue in the complex case? One clue is given by the existence, in l-adic cohomology, of an increasing filtration, the weight filtration $W$, for which the $i$-th quotient $W_{i} / W_{i-1}$ is a subquotient of the cohomology of a projective nonsingular variety. We hence expect in the complex case a filtration $W$ such that the $i$-th quotient has a Hodge decomposition of weight $i$. Another clue, coming from works of Griffiths and Grothendieck, is that the Hodge filtration is more important than the Hodge decomposition. Both clues force the definition of mixed Hodge structures, suggest that they form an abelian category, and suggest also how to construct them.

Raussen and Skau: What about the Langlands program? Have you been involved in it?

Deligne: I have been very interested in it, but I have contributed very little. I have only done some work on $G L(2)$, the linear group in two variables. I tried to understand things. A somewhat remote application of the Weil conjecture has been used in Ngo's recent proof of what is called the funda- mental lemma. I didn't do a lot of work myself, though I had a lot of interest in the Langlands program.

\section{French, American, and Russian Mathematics}

Raussen and Skau: You have already told us about the two institutions you mainly have worked for, namely the IHÉS in Paris and then, since 1984, the IAS in Princeton. It would be interesting for us to hear what your motives were for leaving IHÉS and moving to Princeton. Moreover, we would like to hear what unites the two institutions and how they differ, in your opinion.

Deligne: One of the reasons I left was that I don't think it's good to spend all of one's life in the same place. Some variation is important. I was hoping to have some contact with Harish-Chandra, who had done some beautiful work in representation theory and automorphic forms. That was a part of the Langlands program that I am very interested in, but unfortunately Harish-Chandra died shortly before I arrived at Princeton.

Another reason was that I had imposed on myself to give seminars, each year on a new subject, at the IHÉS in Bures. That became a little too much. I was not really able to both give the seminars and to write them down, so I did not impose the same obligation on myself after I came to Princeton. These are the main reasons why I left the IHÉS for IAS in Princeton.

Concerning the difference between the two institutions, I would say that the Institute for Advanced Study is older, bigger, and more stable. Both are very similar in the way that there are many young visitors who come there. So they are not places where you can fall asleep since you will always be in contact with young people who will tell you that you are not as good as you think you are.

In both places there are physicists, but I think the contact with them was more fruitful for me in Princeton than it was in Bures. In Princeton, there have been common seminars. One year was very intense, with both mathematicians and physicists participating. This was due mainly to the presence of Edward Witten. He has received the Fields Medal even though he is a physicist. When Witten asks me questions, it's always very interesting to try to answer them, but it can be frustrating as well.

Princeton is also bigger in the sense that it has not only math and physics, but also the School of Historical Studies and the School of Social Sciences. There is no real scientific interaction with these schools, but it is pleasant to be able to go and hear a lecture about, for instance, ancient China. One good feature about Bures which you do not have in Princeton is the following: In Bures, the cafeteria is too small. So you sit where you can and you don't get to choose the people you are sitting with. I was often sitting next to an analyst or a physicist, and such random informal interactions are very 
useful. In Princeton, there is one table for the mathematicians, another for the astronomers, the ordinary physicists, and so on. You will not be told to go away if you sit down at the wrong table, but still there is segregation.

The Institute for Advanced Study has a big endowment, while the IHÉS had none, at least when I was there. This didn't affect the scientific life. Sometimes it created instability, but the administration was usually able to hide the difficulties from us.

Raussen and Skau: Apart from your connections with French and U.S. mathematics, you have also had a very close contact with Russian mathematics for a long time, even from long before the fall of the Iron Curtain. In fact, your wife is the daughter of a Russian mathematician. How did your contact with Russian mathematics develop?

Deligne: Grothendieck or Serre told Manin, who was in Moscow at the time, that I had done some interesting work. The Academy [Russian Academy of Sciences] invited me to a conference for I. M. Vinogradov, a terribly anti-Semitic person, by the way. I came to Russia, and I found a beautiful culture for mathematics. At that time mathematics was one of the few subjects where the Communist Party could not meddle, as it did not understand it at all, and this turned it into a space of freedom.

We would go to somebody's home and sit by the kitchen table to discuss mathematics over a cup of tea. I fell in love with the atmosphere and this enthusiasm for mathematics. Moreover, Russian mathematics was one of the best in the world at that time. Today there are still good mathematicians in Russia, but there has been a catastrophic emigration. Furthermore, among those wanting to stay, many need to spend at least half of the time abroad, just to make a living.

Raussen and Skau: You mentioned Vinogradov and his anti-Semitism. You talked to somebody and asked whether he was invited?

Deligne: It was Piatetskii-Shapiro. I was completely ignorant. I had a long discussion with him. For me it was obvious that someone like him should be invited by Vinogradov, but I was told that that was not the case.

After this introduction to Russian mathematics, I still have some nostalgia for the beautiful memories of being in Moscow and speaking with Yuri Manin and Sergey Bernstein or being at the Gelfand seminar. There was a tradition, which still exists, of a strong connection between the university and the secondary education. People like Andrey Kolmogorov had a big interest in secondary education (perhaps not always for the best).

They have also the tradition of Olympiads, and they are very good at detecting promising people in mathematics early on in order to help them. The culture of seminars is in danger because it's important that the head of the seminars is working full-time in Moscow, and that is not always the case. There is a whole culture which I think is important to preserve. That is the reason why I used half of the Balzan Prize to try to help young Russian mathematicians.

Raussen and Skau: That was by a contest that you arranged.

Deligne: Yes. The system is falling apart at the top because there is no money to keep people, but the infrastructure was so good that the system continues to produce very good young mathematicians. One has to try to help them and make it possible for them to stay somewhat longer in Russia so that the tradition can continue.

\section{Competition and Collaboration in Mathematics}

Raussen and Skau: Some scientists and mathematicians are very much driven by the aim to be the first to make major discoveries. That seems not to be your main driving force?

Deligne: No. I don't care at all.

Raussen and Skau: Do you have some comments on this culture in general?

Deligne: For Grothendieck it was very clear: he once told me that mathematics is not a competitive sport. Mathematicians are different, and some will want to be the first, especially if they are working on very specific and difficult questions. For me it's more important to create tools and to understand the general picture. I think mathematics is much more a collective enterprise of long duration. In contrast to what happens in physics and biology, mathematical articles have long and useful lives. For instance, the automatic evaluation of people using bibliographic criteria is particularly perverse in mathematics, because those evaluation methods take account only of papers published during the last three or five years. This does not make sense in mathematics. In a typical paper of mine, I think at least half of the papers cited can be twenty to thirty years old. Some will even be two hundred years old.

Raussen and Skau: You like to write letters to other mathematicians?

Deligne: Yes. Writing a paper takes a lot of time. Writing it is very useful, to have everything put together in a correct way, and one learns a lot doing so, but it's also somewhat painful. So in the beginning of forming ideas, I find it very convenient to write a letter. I send it, but often it is really a letter to myself. Because I don't have to dwell on things the recipient knows about, some short-cuts will be all right. Sometimes the letter, or a copy of it, will stay in a drawer for some years, but it preserves ideas, and when I eventually write a paper, it serves as a blueprint.

Raussen and Skau: When you write a letter to someone and that person comes up with additional ideas, will that result in a joint paper? 
Deligne: That can happen. Quite a lot of my papers are by me alone, and some are joint work with people having the same ideas. It is better to make a joint paper than having to wonder who did what. There are a few cases of genuine collaborations where different people have brought different intuitions. This was the case with George Lusztig. Lusztig had the whole picture of how to use l-adic cohomology for group representations, but he did not know the techniques. I knew the technical aspect of $l$-adic cohomology, and I could give him the tools he needed. That was real collaboration.

A joint paper with Morgan, Griffiths, and Sullivan was also a genuine collaboration. Also with Bernstein, Beilinson, and Gabber: we put together our different understandings

\section{Work Style, Pictures, and Even Dreams}

Raussen and Skau: Your CV shows that you haven't taught big classes of students a lot. So, in a sense, you are one of the few full-time researchers in mathematics.

Deligne: Yes. And I find myself very lucky to have been in this position. I never had to teach. I like very much to speak with people. In the two institutions where I have worked young people come to speak with me. Sometimes I answer their questions, but more often I ask them counter-questions that sometimes are interesting, too. So this aspect of teaching with one-to-one contact, trying to give useful information and learning in the process, is important to me.

I suspect it must be very painful to teach people who are not interested, but are forced to learn math because they need the grade to do something else. I would find that repulsive.

Raussen and Skau: What about your mathematical work style? Are you most often guided by examples, specific problems and computations, or are you rather surveying the landscape and looking for connections?

Deligne: First I need to get some general picture of what should be true, what should be accessible, and what tools can be used. When I read papers I will not usually remember the details of the proofs, but I will remember which tools were used. It is important to be able to guess what is true and what is false in order not to do completely useless work. I don't remember statements that are proved, but rather I try to keep a collection of pictures in my mind-more than one picture, all false but in different ways, and knowing in which way they are false. For a number of subjects, if a picture tells me that something should be true, I take it for granted and will come back to the question later on.

Raussen and Skau: What kind of pictures do you have of these very abstract objects?

Deligne: Sometimes very simple things! For instance, suppose I have an algebraic variety, and hyperplane sections, and I want to understand how they are related, by looking at a pencil of hyperplane sections. The picture is very simple. I draw it in my mind something like a circle in the plane and a moving line that sweeps it. Then I know how this picture is false: the variety is not one-dimensional, but higher-dimensional, and when the hyperplane section degenerates, it is not just two intersection points coming together. The local picture is more complicated, like a conic that becomes a quadratic cone. These are simple pictures put together.

When I have a map from some space to another I can study properties it has. Pictures can then convince me that it is a smooth map. Besides having a collection of pictures, I also have a collection of simple counter-examples, and statements that I hope to be true have to be checked against both the pictures and the counter-examples.

Raussen and Skau: So you think more in geometric pictures than algebraically?

Deligne: Yes.

Raussen and Skau: Some mathematicians say that good conjectures, or even good dreams, are at least as important as good theorems. Would you agree?

Deligne: Absolutely. The Weil conjectures, for instance, have created a lot of work. Part of the conjecture was the existence of a cohomology theory for algebraic systems with some properties. This was a vague question, but that is all right. It took over twenty years of work, even a little more, in order to really get a handle on it. Another example of a dream is the Langlands program, which has involved many people over fifty years, and we have now only a slightly better grasp of what is happening.

Another example is the philosophy of motives of Grothendieck, about which very little is proved. There are a number of variants taking care of some of the ingredients. Sometimes, such a variant can be used to make actual proofs, but more often the philosophy is used to guess what happens, and then one tries to prove it in another way. These are examples of dreams or conjectures that are much more important than specific theorems.

Raussen and Skau: Have you had a "Poincaré moment" at some time in your career where you, in a flash, saw the solution of a problem you had worked on for a long time?

Deligne: The closest I have been to such a moment must have been while working on the Weil conjecture when I understood that perhaps there was a path using Rankin against Grothendieck. It took a few weeks after that before it really worked, so it was a rather slow development. Perhaps also for the definition of mixed Hodge structures, but also in this case, it was a progressive process. So it was not a complete solution in a flash.

Raussen and Skau: When you look back on fifty years of doing mathematics, how have your work 
and your work style changed over the years? Do you work as persistently as you did in your early years?

Deligne: I am not as strong as I was earlier, in the sense that I cannot work as long or as intensively as I did. I think I have lost some of my imagination, but I have much more technique that can act as a substitute to some extent. Also the fact that I have contact with many people gives me access to some of the imagination I am lacking myself. So when I bring my technique to bear, the work can be useful, but I'm not the same as when I was thirty.

Raussen and Skau: You have retired from your professorship at IAS rather early...

Deligne: Yes, but that's purely formal. It means I receive retirement money instead of a salary, and no school meetings for choosing next year's members. So that's all for the best. It gives me more time for doing mathematics.

\section{Hopes for the Future}

Raussen and Skau: When you look at the development of algebraic geometry, number theory, and the fields that are close to your heart, are there any problems or areas where you would like to see progress soon? What would be particularly significant, in your opinion?

Deligne: Whether or not it's within reach in ten years, I have absolutely no idea; as it should be... But I would very much like to see progress in our understanding of motives. Which path to take and what are the correct questions, is very much in the air. Grothendieck's program relied on proving the existence of algebraic cycles with some properties. To me this looks hopeless, but I may be wrong.

The other kind of question for which I would really like to see some progress is connected with the Langlands program, but that is a very long story...

In yet another direction, physicists regularly come up with unexpected conjectures, most often using completely illegal tools. But, so far, whenever they have made a prediction, for instance a numerical prediction on the number of curves with certain properties on some surface-and these are big numbers, in the millions perhaps-they were right! Sometimes previous computations by mathematicians were not in accordance with what the physicists were predicting, but the physicists were right. They have put their fingers on something really interesting, but we are, so far, unable to capture their intuition. Sometimes they make a prediction, and we work out a very clumsy proof without real understanding. That is not how it should be. In one of the seminar programs that we had with the physicists at IAS, my wish was not to have to rely on Ed Witten but instead to be able to make conjectures myself. I failed! I did not understand enough of their picture to be able to do that, so I still have to rely on Witten to tell me what should be interesting.
Raussen and Skau: What about the Hodge conjecture?

Deligne: For me, this is a part of the story of motives, and it is not crucial whether it is true or false. If it is true, that's very good, and it solves a large part of the problem of constructing motives in a reasonable way. If one can find another purely algebraic notion of cycles for which the analogue of the Hodge conjecture holds, and there are a number of candidates, this will serve the same purpose, and I would be as happy as if the Hodge conjecture were proved. For me it is motives, not Hodge, that is crucial.

\section{Private Interests-and an Old Story}

Raussen and Skau: We have the habit of ending these interviews by asking questions that are outside of mathematics. Could you tell us a little bit about your private interests outside your profession? We know about your interest in nature and in gardening, for example.

Deligne: These are my main interests. I find the earth and nature so beautiful. I don't like just to go and have a look at a scenery. If you really want to enjoy the view from a mountain, you have to climb it on foot. Similarly, to see nature, you have to walk. As in mathematics, in order to take pleasure in nature-and nature is a beautiful source of pleasure-one has to do some work.

I like to bicycle because that's also a way to look around. When distances are a little bigger than what is convenient on foot, this is another way of enjoying nature.

Raussen and Skau: We heard that you also build igloos?

Deligne: Yes. Unfortunately, there's not enough snow every year and even when there is, snow can be tricky. If it's too powdery, it's impossible to do anything; likewise if it's too crusty and icy. So there is maybe just one day, or a few hours each year when building an igloo is possible, and one has to be willing to do the work of packing the ice and putting the construction together.

Raussen and Skau: And then you sleep in it?

Deligne: And then I sleep in the igloo, of course.

Raussen and Skau: You have to tell us what happened when you were a little child.

Deligne: Yes. I was in Belgium at the seaside for Christmas, and there was much snow. My brother and sister, who are much older than me, had the nice idea to build an igloo. I was a little bit in the way. But then they decided I might be useful for one thing: if they grabbed me by my hands and feet, I could be used to pack the snow.

Raussen and Skau: Thank you very much for granting us this interview. These thanks come also on behalf of the Norwegian, the Danish, and the European mathematical societies that we represent. Thank you very much!

Deligne: Thank you. 